\title{
How can the personal discovery process evolve personalized medicine? An interview with Laura Towart
}

\author{
Laura A Towart ${ }^{*, 1}$ \\ ${ }^{1}$ My Personal Therapeutics, Open Cell London, United Kingdom \\ *Author for correspondence: laura@mypersonaltherapeutics.com
}

Laura is the Founder and CEO of My Personal Therapeutics, a London-based digital health company offering the most advanced personalized cancer therapeutics. She is also the Founder and former CEO of Celmatix, a leader in diagnostics and predictive analytics for female infertility and women's health. She is a graduate of the Weill Cornell Graduate School of Medical Sciences and Memorial Sloan Kettering Cancer Center's Doctoral program and received a Certificate in Bioinformatics. She holds a BS/BA in Biology/English from The George Washington University. Besides being passionate about personalized medicine, she enjoys exploring the world with her children Julian, Valentina and Delphine.

First draft submitted: 28 June 2019; Accepted for publication: 11 July 2019; Published online: 13 September 2019

Keywords: artificial intelligence $\bullet$ big data $\bullet$ cancer $\bullet$ cancer research $\bullet$ colorectal cancer $\bullet$ genomics $\bullet$ health artificial intelligence $\bullet$ machine learning $\bullet$ personalized medicine $\bullet$ precision cancer $\bullet$ precision medicine

\section{Please introduce yourself to our readers \& tell them about your career to date}

Hello, I am Laura Towart, Founder and CEO of My Personal Therapeutics. Early in my career, I trained as a scientist and was a fellow at the Tri-Insitutional PHD program at Weill Cornell Graduate School of Medical Sciences/Memorial Sloan Kettering and Rockefeller Institute (NY, USA). It was the advent of the genomics era and my room mate and I developed the idea to create a genetic test for female infertility in women with idiopathic infertility. In 2008, I left my PhD program and co-founded Celmatix (NY, USA). Since then, we succeeded in creating our genetic test, called Fertilome, and identified important targets for pharmaceutical development of women's health products. I was the founding CEO of Celmatix and I currently serve as a Director. In 2012, I learned about a technology in development at the Icahn School of Medicine at Mt Sinai Medical Center called the Personal Discovery Process (PDP). I was excited about the potential of PDP to transform personalized medicine for cancer, and also for any disease of genetic origin. I began to work with Mount Sinai on commercialization of PDP. This year, I licensed the technology in partnership with Mount Sinai and founded My Personal Therapeutics (London, UK). We licensed the technology in April and are currently developing our infrastructure and introducing our offerings to patients worldwide.

What does your current role as the Founder of My Personal Therapeutics involve?

My role as the Founder and CEO is to make the Personal Discovery Process available to patients and to build a community of people around the technology. I have been focused on recruiting a stellar team. I have hired a Chief Scientific Officer, Dr Santiago Nahuel Villegas and Senior Scientists, a Lab Manager and we continue to expand. I have been meeting with oncologists and introducing PDP to stakeholders around the world. We are building a state of the art laboratory in central London. Along with managing the development of the My Personal Therapeutics app. The aim of the app is to educate patients on PDP, help them understand our innovative offering and keep them informed of where they are in the process of their personalized treatment. Our ultimate goal is to build our artificial intelligence (AI)/machine learning (ML) driven product TuMatch and so everything I do it with this aim in mind. 


\section{How does The PDP create treatment recommendations for individual cancer patients?}

The PDP starts with the patient and their oncologist. Either the patient or oncologist contacts My Personal Therapeutics and we then determine if PDP is appropriate for them. If they are a candidate they will have their tumor biopsied and sequenced at their point of care. The sequence information (including blood sequence) would then be transferred to My Personal Therapeutics via the cloud. Our process starts with a very deep analysis of the tumor whole exome. We determine the genetic drivers of the tumor, including known oncogenes and other genes that may be driving tumorigenesis. We select up to 15 genetic mutations and build them into personalized fruit fly avatars. Depending on the type of cancer, we grow up to half a million fruit fly avatars. The avatars will develop a very genetically similar tumor to the patient. If the patient has colorectal cancer, we are able to micro-inject the tumor construct so that the tumor will grow in the area of the fly corresponding to the colorectum, as similar to the patient as possible. Using robotics, we screen every US FDA approved drug in up to three/four combinations, and we screen (the fly avatars) to see which of those combinations rescues the fly from death. We are then able to make a treatment recommendation based on this information. For every patient so far, there has been more than one combination predicted from the flies. All combinations will include one chemo drug and either one, two or three nonchemo drugs. The combinations will be less toxic, less expensive and overall more targeted to the patient's tumor.

\section{How do you use Al at My Personal Therapeutics to optimize the PDP?}

Patients with similar tumor profiles will respond to the same treatment recommendations. Therefore, we use AI to predict drug recommendations depending on tumor genomic profile. We are developing TuMatch, an AI-powered service, which will suggest treatment options for a patient's specific tumor genetics. Based on Mount Sinai's results, we expect that data from a few hundred patients per tumor type will be needed to operationalize TuMatch. To feed TuMatch, we offer two types of drug screening: PDP, which evaluates $>1500$ drug treatments over up to 5 months and targeted PDP, which evaluates approximately 200 drug treatments in up to 4 months.

We will develop TuMatch for all tumor types with a significant genetic cause. We are initially focusing on colorectal cancer as it is largely unresponsive to immunotherapy and manifest deep genetically driven pockets of resistance. We hope to be able to offer TuMatch for colorectal cancer and gastrointestinal cancer, by early 2020, with other tumor types later. TuMatch will enable rapid and affordable personalized drug treatment recommendations.

\section{What do you feel are the biggest challenges for PDP \& applying this in clinic?}

The biggest challenge right now is the time it takes to get to treatment recommendations. Until we complete the development of TuMatch it will take up to 5 months for a patient to receive their personalized treatment recommendations. We are trying to decrease this timeline by innovating at each step of our process and integrate machine learning and AI where possible. We are now reliant on building fly avatars, which is a complex, labor intensive process.

To make up for this time, we recommend patients to start treatment with the standard of care and have a PDP model built as an insurance policy in case they develop resistance or do not respond to front-line treatments. Ideally, the patient or oncologist would approach My Personal therapeutics before the patient becomes resistant or if they have been found to be prone to metastases. However, this is idealistic and does not always happen that way.

\section{In what way does the data provided by Genomics England help with your research?}

Usually when we receive patient genomic information, it is in the form of whole exome sequence data. However, Genomics England provide us with whole genome sequence information, which enhances our ability to select driver mutations. We are able to scan Genomics England's database and intelligently select which mutational profiles we do not have and then build and screen avatars to populate and enrich our dataset. Utilizing databases such as Genomics England's, we can build avatars for the most common tumor genomes and screen the FDA-approved drug library to build our database and expedite the process for incoming patients. However, our ability to do that depends on our funding, so we have also applied for number of grants.

\section{What do you think the future of personalized medicine looks like as technology continues to evolve \& how does the Personal Discovery Process fit into this?}

In the future, I imagine there will be personalized drug cocktails for many genetic diseases, including cancer. Other companies are also working on this and are utilizing AI to identify targets in various datasets. For example, once My 
Personal Therapeutics identifies personalized drug treatment recommendations for patients, we want to understand how that drug combination is uniquely overcoming mechanisms of resistance for tumors. We will work with centers of excellence such as Medicines Discovery Catapult to gain an understanding of how these drugs are targeting the tumor complex and how we can potentially optimize the drug combinations. Potentially, we could design new drugs so that rather than taking three drugs the patient could take one that is optimized.

\section{Financial \& competing interests disclosure}

The interviewer is a shareholder and employee at My Personal Therapeutics. The interviewee has no other relevant affiliations or financial involvement with any organization or entity with a financial interest in or financial conflict with the subject matter or materials discussed in the manuscript apart from those disclosed.

No writing assistance was utilized in the production of this manuscript.

\section{Disclaimer}

The opinions expressed in this article are those of the author and do not necessarily reflect the views of Future Medicine Ltd. 